(RESEARCH ARTICLE)

\title{
Left-handers, retrained left-handers and right-handers: A comparative study
}

\author{
Makashvili Malkhaz ${ }^{1,}{ }^{*}$, Kokrashvili Elene ${ }^{1}$, Kopadze Tamar ${ }^{2}$, Enukidze Guram ${ }^{1}$ and Abuladze Nikoloz ${ }^{1}$ \\ ${ }^{1}$ Center for psycho-physiological research, Ilia State University, Tbilisi, Georgia. \\ ${ }^{2}$ European University, Tbilisi, Georgia.
}

Publication history: Received on 24 June 2020; revised on 05 July 2020; accepted on 07 July 2020

Article DOI: https://doi.org/10.30574/wjarr.2020.7.1.0227

\begin{abstract}
Present study was aimed at further extension of the scientific data concerning the influence of retraining in left-handers as well as concerning some disabilities ascribed to the left-handedness. Study participants filled in the questionnaire about the incidence of fears and depression, bedwetting and stuttering, spatial confusion, complaints on weak memory and attention. Responses were analyzed regarding the handedness of the respondents, qualified as left-hander, righthander and retrained left-hander. The number of reports on bedwetting and slowness in writing, as well as on spatial left/right confusion was found higher in left-handers as compared to right-handers. The number of reports on fears was found higher in retrained left-handers. Left-handers, retrained left-handers and right-handers did not differ in the number of reports on stuttering, depression, week memory and not sustained attention. Mental rotation task performance was studied in another group of left-handed and right-handed participants. Handedness was determined by the test on hand performance. Left-handers were found to make more mistakes in mental rotation task performance as compared to right-handers. We conclude that expectancy of bedwetting, slowness in writing and mental spatial left/right confusion is higher in left-handers as compared to right-handers. Retraining of left-handers is suggested to cause fears, however it is not related to stuttering, depression, slowness in reading and writing, worsening of memory and attention. Stuttering is equally expectable among left-handers, retrained left-handers and right-handers.
\end{abstract}

Keywords: Left-handers; Retraining; Spatial orientation; Fears; Stuttering; Bedwetting; Writing

\section{Introduction}

The term "Retraining" denotes the conversion of left-handers to the writing with a right hand or complete shifting to the use of the right hand instead of the left limb in both, writing as well as everyday manipulations, such as eating, use of brush or comb, drawing etc. [1]. Retraining was widely used in the Western World in 19th century and in the first decades of 20 th century. Scientists, physicians and educators strongly advised to retrain left-handed children [see 2 for review]. Some facts of retraining by Western school teachers were registered in the second half of 20th century [3] and nowadays, retraining is practiced in Asia and Africa [2,4] as well as in Georgia [5]. The idea about the necessity of retraining is based on the social and cultural discrimination of left-handers [6,7], as well as on the supposed correlation of left-handedness to the various types of disabilities [1]. Explaining their attempts to retrain pupils, some teachers refer to the school tradition of writing with the right hand as well as to the tradition of Orthodox Church to make a sign of the cross with the right hand [5], while both explanations are irrelevant in fact. Retraining may have negative life-long consequences, especially if retraining methods are coercive. In personal communications left-handers witness stuttering, slaw reading and writing, bedwetting, nail biting, anxiety, disorientation in space after being forcedly retrained [2]. However, scientific data still are not clear enough to claim, that these symptoms develop after retraining and are not, in fact, common to left-handers, no matter are they retrained or not. Some theories [8] and research data [9] suggest, that left-handers are generally disadvantaged relative to right-handers. Starting in early decades of the twentieth century, there are two contradictory ideas about the influence of retraining on left-handers. Some scientists argued, that retraining may result in stuttering while others supposed, that stuttering which may have come on during retraining, is not the result of this procedure, but rather a trait, associated to the left-handedness [see 1 for review].

\footnotetext{
* Corresponding author: Makashvili Malkhaz
}

Copyright (C) 2020 Author(s) retain the copyright of this article. This article is published under the terms of the Creative Commons Attribution Liscense 4.0. 
Left-handedness was considered a manifestation of atypical cerebral lateralization, leading to developmental disabilities, in particular, to stuttering [10-12]. Authors [13] reported on a higher frequency of stuttering in left-handers as compared to right-handers in the large group of 9591 male subjects between 17 and 24 years of age. Some other authors however, reported, that stutterers had neither a greater incidence of left-handedness than is usually reported for the general population, nor demonstrated less consistency than fluent speakers in hand preference [14-16 ]. The proportion of right and left-handers in the group of more than 1000 stutterers was found similar to that reported in the general population [17]. According to other authors, the incidence of left-handedness in stuttering children was not significantly different from normally fluent children. The difference between the left and right-handed subjects was not statistically significant in variables of stuttering severity as well [18]. In sum, further research is necessary to learn more about the results of retraining in left-handers. Another question concerning the human handedness is if left-handers have worth spatial ability as compared to right-handers. Right-handed 5 years old children scored higher than lefthanders in the tasks on identification of the right and left parts of their own bodies as well as in the tasks on identifying the right and left parts of the viewed model figures [19]. Left-handed mail participants were found performing the Bergen left-right discrimination better than right-hander males, however this was sex-depended effect while the effect of handedness was found insignificant in the total number of male and female participants [20]. No difference in the left right discrimination between the left-handed and right-handed people was found in other studies as well [21-24].

Present study was aimed at further extension of the scientific data concerning the influence of retraining in left-handers as well as concerning spatial confusion and some disabilities ascribed to the left-handedness.

\section{Material and methods}

Left-handed and right-handed people, aged 23-40, mean age 31.5, of both sexes participated in the study. Two series of the study have been conducted. In the first series the "Survio" program was used to engage people in the study. The questionnaire was disseminated through Facebook and by personal internet contacts. Instruction was as following: This questionnaire is designed to collect the data on the health status of local population. This is anonymous questionnaire and we ask for your kind openness. You are requested to click "yes" or "no" in response to the statements as following: A. I am right-hander and I use my right hand in writing and everyday activities such as holding fork, drawing, using computer mouse, using scissors, throwing ball], B. I have never been left-handed and retrained to the right-hand activity, C. I am left-hander and I use my left hand in writing and everyday activities (such as holding fork, drawing, using computer mouse, using scissors, throwing ball), D. I am retrained left hander, forced to use my right hand instead of the left hand in writing, however I use my left hand for everyday activities (such as holding fork, drawing, using computer mouse, using scissors, throwing ball), E. I am retrained left-hander, I use my right hand for writing, as well as for most [or all] of everyday activities (such as holding fork, drawing, using computer mouse, using scissors, throwing ball), F. I experience, or have been experiencing in school years: 1- stuttering, 2- bedwetting, 3- anxiety, 4- depression 5-fears, 6- week memory, 7 -slowness in reading, 8 -slowness in writing, 9- not sustained attention, 10- confusion in left/right spatial orientation. Respondents had to click "yes" or "no" in response to the each statement in the list. Responses were divided into two groups: Group R: Responses of the right-handers, criteria for inclusion: answer "Yes" to the questions A and B and answer "No" to the questions C, D, E and Group L: Responses of the left-handers, criteria for inclusion: answer "No" to the questions A and B and answer "Yes" to the questions C and D and "No" to the question E. Responses with positive answer to E were excluded from the study. In turn, Group L was divided in 2 subgroups: LLnon retrained left-handers answering "Yes" to the question C and "No" to the question D and LR - retrained left-handers, answering "Yes" to the question D and "No" to the question C. Out of the total of 962 responses, 369 fall into the L, 185 into the LR and 408 - into the R.

In the second series spatial ability of another group of right-handed (R2) and left-handed, not retrained (LL2) participants was examined. Hand performance index R-L/R+L was calculated. Out of the total of 104 participants 52 were identified as left-handers (handedness index range -0.6 to -1 ), and the rest 52 were identified as right-handers (handedness index range +0.8 to +1 ). Four pen and paper tasks on spatial left/right orientation were presented to each participant in vis-a-vie situation. Task N1: Contour drawing of a human figure, facing the beholder, was presented to the participant. Instruction: This figure is looking at you, please point to its left hand. Task N2: Contour drawing of a human figure, with its back to the beholder, was presented to the participant. Instruction: This figure turns its back to you, point to its left hand. Task N3. Two contour drawings of a human figure, one - A with its back to the beholder, another - B, facing the beholder, the latter holding the flag in one hand. Instruction: Look, the A is turned with its back to you and it holds a flag in the hand. If B turns like A, where would the flag be placed? draw the flag on the A. Tasks 1-3 belong to socalled Mental rotation task, widely accepted for evaluation of spatial left/right orientation. Task 4. Participants were instructed to draw a line. The line direction was dictated by the investigator in the sequence such as: move forward, take right, move downward, take left, move downward, take right, move forward, and take right. Participants were requested to repeat each test until correct answer. The number of mistakes in the each test performance was calculated. 
All methodological approaches, used in the study, have been approved by the Ethical Committee of Psychologists at the Ilia University.

Chi square for comparison of groups using categorial data was used to analyze the results of the first series of the study. An independent samples t-test was conducted for statistical analysis of the results in the second series of the study.

\section{Results and discussion}

See Table 1 for the results of LL and LR comparative study.

Table 1 Frequency (upper raw) and percent (lower raw) of symptoms in the total number of respondents, in LL and LR.

\begin{tabular}{llllllllllll}
\hline $\begin{array}{l}\text { No. } \\
\text { participants }\end{array}$ & of & $\mathbf{1}$ & $\mathbf{2}$ & $\mathbf{3}$ & $\mathbf{4}$ & $\mathbf{5}$ & $\mathbf{6}$ & $\mathbf{7}$ & $\mathbf{8}$ & $\mathbf{9}$ & $\mathbf{1 0}$ \\
\hline LL & 44 & 19 & 73 & 72 & 80 & 56 & 42 & 52 & 86 & 70 \\
369 & $11.9 \%$ & $5.1 \%$ & $29.8 \%$ & $19.5 \%$ & $21.7 \%$ & $15.2 \%$ & $11.4 \%$ & $14.1 \%$ & $23.3 \%$ & $19 \%$ \\
LR & 30 & 11 & 56 & 48 & 51 & 30 & 23 & 34 & 30 & 52 \\
185 & $16.2 \%$ & $5.9 \%$ & $30.3 \%$ & $23.9 \%$ & $27.6 \%$ & $16.2 \%$ & $12.4 \%$ & $18.4 \%$ & $16.2 \%$ & $28.1 \%$
\end{tabular}

1- stuttering, 2- bed wetting, 3- anxiety, 4- depression 5-fears, 6- weak memory, 7- slowness in reading, 8-slowness in writing, 9- lack of attention, 10- left/right confusion

As compared to LL, higher frequency of positive answers to the questions 5 and 10 was registered in LR (see Figure 1).

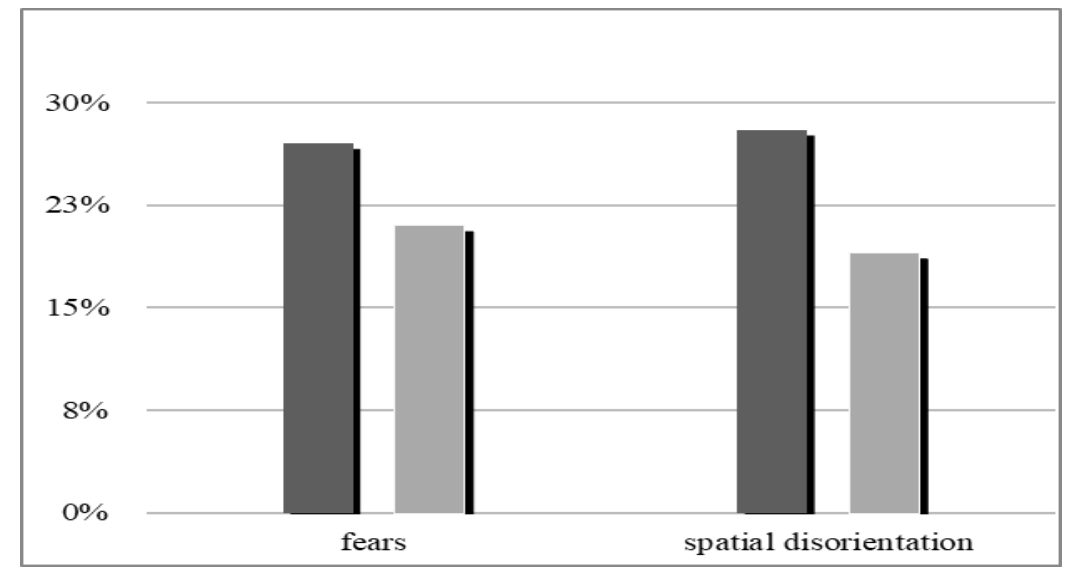

Figure 1 The frequency of fears and spatial left/right confusion in LR (black column) and LL (grey column)

A Chi-square test of independence revealed that significantly more LR (27.6\%) as compared to LL (21.7\%) witnessed to having fears, $\chi^{2}(1, N=554)=7.59, p=.006$ as well as left/right confusion in spatial orientation $(28.1 \%$ and $19 \%$ respectively), $\chi^{2}(1, N=554)=5.99, p=.014$.

No statistically reliable difference in the rate of positive responses to the questions regarding the incidence of stuttering, bed wetting, anxiety, depression, weak memory, slowness in reading and writing and weak attention between the LL and LR was found.

See Table 2 for the results of LL+LR and R comparative study. 
Table 2 Frequency (upper raw) and percent (lower raw) of symptoms in the total number of respondents, in LL+LR and $\mathrm{R}$.

\begin{tabular}{lllllllllll}
\hline N of participants & $\mathbf{1}$ & $\mathbf{2}$ & $\mathbf{3}$ & $\mathbf{4}$ & $\mathbf{5}$ & $\mathbf{6}$ & $\mathbf{7}$ & $\mathbf{8}$ & $\mathbf{9}$ & $\mathbf{1 0}$ \\
\hline LL+LR & 74 & 30 & 129 & 120 & 131 & 86 & 65 & 86 & 116 & 122 \\
554 & $13.4 \%$ & $5.4 \%$ & $23.3 \%$ & $21.7 \%$ & $23.6 \%$ & $15.5 \%$ & $11.7 \%$ & $15.5 \%$ & $20.9 \%$ & $22 \%$ \\
$\mathrm{R}$ & 45 & 10 & 152 & 151 & 192 & 124 & 39 & 38 & 207 & 70 \\
408 & $11 \%$ & $2.5 \%$ & $37.3 \%$ & $37 \%$ & $47.1 \%$ & $30.4 \%$ & $9.6 \%$ & $9.3 \%$ & $50.7 \%$ & $9.1 \%$
\end{tabular}

1-stuttering, 2- bed wetting, 3- anxiety, 4- depression 5-fears, 6- weak memory, 7-slowness in reading, 8-slowness in writing, 9- lack of attention, 10- left/right confusion

The frequency of positive answers to the questions regarding bed-wetting, slowness in writing and spatial left/right confusion was higher in LL+LR as compared to the R (see Figure 2).

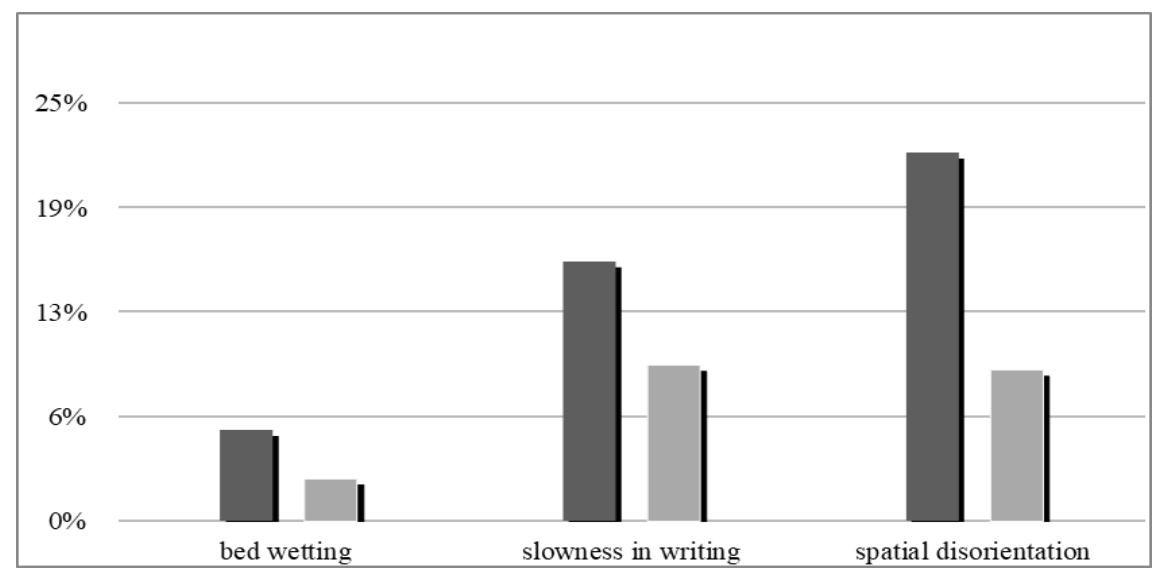

Figure 2 The frequency of bedwetting, slowness in writing and spatial left/right confusion in LL (black column) and R (gray column).

The frequency of positive answers to the questions regarding bed-wetting, slowness in writing and left/right confusion was higher in LL+LR (5.4\%, 15,5\% and $22 \%$ respectively) as compared to the $\mathrm{R}(2.5 \%, 9.3 \%$ and $9 \%$ respectively).

Statistical analysis confirmed the prevalence of bedwetting $\chi^{2}(1, N=962)=5.18, p=.023$, slowness in writing $\chi^{2}(1$, $N=962)=8.06, p=.005$. and left/right confusion $\chi^{2}(1, N=962)=28.574, p=.001$ in the LL+LR as opposed to R.

See Table 3 for the results of Mental rotation task performance. As compared to R2 (M = 0.04, SD = 0.19]) LL2 (M = 0.21, $\mathrm{SD}=0.41$ ) demonstrated statistically significant more errors $(102)=2.738, \mathrm{p}=.008$.

Table 3 T-test results, comparing Task 1 performance in LL and R2

\begin{tabular}{lcccccc}
\hline participants & $\mathbf{n}$ & Mean & SD & Df & t & p \\
\hline LL2 & 52 & 0.21 & 0.41 & 102 & 2.738 & 0.008 \\
R2 & 52 & 0.04 & 0.19 & & & \\
\hline
\end{tabular}

Similar results were observed in the Task N2 performance (see Table 4). LL2 (M = 0.12, SD = 0.32) compared to the R2 $(\mathrm{M}=0, \mathrm{SD}=0)$, where more prone to errors $\mathrm{t}(102)=2.579, \mathrm{p}=.013$. 
Table 4 T-test results, comparing Task 2 performance in LL2 and R2

\begin{tabular}{lllllll}
\hline Participants & $\mathbf{n}$ & Mean & SD & Df & t & p \\
\hline LL2 & 52 & 0.12 & 0.32 & 102 & 2.579 & 0.013 \\
R2 & 52 & 0 & 0 & & & \\
\hline
\end{tabular}

No statistical difference in Task N3 and Task N4 performance between the LL2 and R2 was observed.

The data obtained suggest, that forced conversion of left-handers to the use of the right hand in writing may lead to fears. Scientific data concerning the negative results of retraining and personal reports of left-handers on their emotional problems due to retraining [2], as well as the results of the current study are strongly convincing, that parents and school teachers should refrain from the retraining of left-handed children. This should be taken into account by parents and teachers in countries, where retraining of left-handers is still practiced. However, according to our study, bedwetting, depression and stuttering, as well as slowness in reading and writing do not result from retraining, as it is suggested by some authors [2]. At the same time, data obtained confirm the suggestion, that stuttering is not a common finding in left-handed individuals [14-18]. However, some symptoms are likely be more common in left-handers than in right-handers. In particular, data obtained suggest that bed-wetting and slowness in writing is more expected in lefthanded individuals as compared to right-handers. Data regarding slaw writing are in accordance to the suggestion, that left-handers write slowly because of poor paper-pen control [25] and that writing is the most difficult school subject for left-handers [26]. As for bedwetting, the data obtained are in accordance to the report on the prevalence of left-handers among the patients with nocturnal enuresis [27]. Complaints about weak memory and attention were of the same rate in left-and right-handers. The data obtained are interesting in the light of the contradiction in research data concerning the correlation between the handedness and attention deficit in ADHD [28, 29]. Left-handers were found to suffer from left/right spatial confusion, as it is apparent from the self-assessment responses of the left-handed participants. The data obtained are in accordance with the report on the prevalence of left-handers over right-handers in self-reports of left/right confusion [30]. Results of the mental rotation task performance confirm, that as compared to right-handers, left-handers have more problems in mental rotation, in particular, in fast understanding of the change in spatial left/right orientation of the observed static visual stimuli - human figures. These data are in accordance with the report on better performance of right-handed children on identifying the right and left parts of the viewed model figures as compared to left-handed coevals [20]. At the same time, left-handed and right-handed participants displayed left/right confusion in task 4 performance - when required to change the direction of line drawing and this task proved equally complicated for both, left-handers as well as right-handers. Task 4 differs from the Tasks 1-3 in that the former requires and is related to the fast left/right orientation during hand movement, rather than mental rotation. Problems in mental spatial rotation, revealed in the present study confirm the suggestion that left-handers may have a special learning needs [30]. It should be underlined, however, that problems in left/right mental rotation, displayed by left-handed participants, disappear after brief training, as long as left-handers displayed worth results in the Tasks 1 and 2, however did not differ from right-handers in the number of mistakes in the Task 3 performance.

The limitation of the current study is that we do not know, which participants had a familial history of handedness and which did not. Therefore we cannot discuss the data obtained with regard to the suggestion, that non-familial lefthandedness is related to particular psychological and developmental problems. The questionnaire used in the current study does not provide information about the quality of stuttering and the data obtained only refer to the rate but not quality of stuttering in study participants. Question concerning bedwetting implied the incidence of this symptom in either period of life - childhood and adulthood and we are not sure about the exact period, participants experienced the bedwetting.

\section{Conclusion}

We conclude that expectancy of bedwetting, slowness in writing and mental spatial left/right confusion is higher in lefthanders as compared to right-handers. Retraining of left-handers is suggested to cause fears, however it is not related to stuttering, depression, slowness in reading and writing, worsening of memory and attention. Stuttering is equally expectable among left-handers, retrained left-handers and right-handers. 


\section{Compliance with ethical standards}

\section{Disclosure of conflict of interest}

Authors do not have a conflict of interests to disclose.

\section{Statement of informed consent}

Informed consent was obtained from all individual participants included in the study.

\section{References}

[1] Kushner HI. (2012). Retraining left-handers and the etiology of stuttering: The rise and fall of an intriguing theory. Laterality, 17(6), 673-693.

[2] Kushner HI. (2017). On the other hand. Left hand, right brain, mental disorder and history. (Johns Hopkins University Press Ed.) Baltimore.

[3] Arrivet D. (2016.) Les entreprises multiplient les produits concus pour les Gauchers. Le Figaro Economie, August, 13.

[4] Singh M, Manjary M and Dellatolas G. (2001). Lateral preferences among Indian school children. Cortex, 37(2), 231-241.

[5] Makashvili M and Taliashvili T. (2009). On the negative attitude towards left-handedness of pupils. ERIC, 1-3.

[6] Kushner HI. (2013). Deficit or Creativity: Cesare Lombroso, Robert Hertz, and the Meanings of Left Handedness. Laterality, 18(4), 416-436.

[7] Laurens VL, Raymond A and Faurie C. (2009). Why are some people left-handed? An evolutionary perspective. Phil. Trans. R. Soc. B 364, 881-894.

[8] Bakan P, Dibb G and Reed P. (1973). Handedness and birth stress. Neuropsychologia, 11, 363-366.

[9] Johnston DW, Nicholls M, Shah M and Shields M. (2009). Nature's Experiment? Handedness and early childhood development. Demography, 46(2) 281-301.

[10] Geschwind N and Behan P. (1982). Left-handedness: association with immune disease, migraine, and developmental learning disorder. Proc. Nat. Acad. Sci, USA, 79, 5097-5100.

[11] Geschwind N and Galaburda AM. (1985). Cerebral lateralization. Biological mechanisms, associations, and pathology: III. A hypothesis and a program for research. Arch. Neurol, 42, 634-654.

[12] Götestam KO. (1990). Left-handedness among students of architecture and music. Percept. Mot. Skills, 70, 13231327.

[13] Dellatolas G, Annesi I, Jallon P, Chavance M and Lellouch J. (1990). An epidemiological reconsideration of the Geschwind-Galaburda theory of cerebral lateralization. Arch. Neurol, 47(7), 778-782.

[14] Records MA, Heimbuch RC and Kidd KK. (1997). Handedness and stuttering: A dead horse?, Journal of Fluency Disorders, 2, 271-282.

[15] Webster WG and Poulos M. (1987). Handedness distributions among adults who stutter. Cortex, 23, 705-708.

[16] Ardila A, Bateman JR, Niño R, Pulido E, Rivera DB and Vanegas CJ. (1994). An epidemiologic study of stuttering. J. Commun. Disord, 27, 37-48.

[17] Foundas A. (2003). Hand preference and footedness: Atypical handedness in developmental stuttering. Stuttering foundation.

[18] Salihović N and Sinanović O. (2000). Stuttering and left-handedness. Med. Arch, 54(3), 173-175.

[19] Rigal R. (1994). Right-left orientation: Development of correct use of right and left terms. Percept. Mot. Skills, 73 , $1259-1278$.

[20] Ofte SH. (2002). Right-left discrimination: Effects of handedness and educational background. Scand. J. Psychol, 43(3), 213-219. 
[21] Jaspers-Fayer F and Peters M. (2005). Hand preference, magical thinking and left right confusion. Laterality, 10, 183-191.

[22] Grewe P, Ohmann HA, Markowitsch HJ and Piefke M. (2014). The Bergen left right discrimination test: practice effects, reliable change indices, and strategic performance in the standard and alternate form with inverted stimuli. Cogn. Process, 15, 159-172.

[23] McKinley J, Dempster and Gormley GJ. (2015). Sorry, I meant the patient's left side': impact of distraction on left right discrimination. Med. Educ, 49, 427-435.

[24] Constant $M$ and Mellet E. (2018). The impact of handedness, sex, and cognitive abilities on left-right discrimination: A behavioral study. Front. Psychol, 9, 405.

[25] Kula P. (2008). Teaching left-handed primary school pupils in Estonia. J. Teacher Educat. Sustain, 9, 58-67.

[26] Ferrara P, Rugeiro A, Diociaiuti L, Paoletti FP, Chiozza ML and Caione,P. (2001). Primary nocturnal enuresis and left-handedness. Scand. J. Urol. Nephrol, 35, 184-185.

[27] Ghanizadeh A. (2012). Lack of association of handedness with inattention and hyperactivity symptoms in ADHD. J. Atten. Disord, 17 (4) 302-307.

[28] Lin H-L and Tsuang H-Ch. (2018). Handedness and Attention Deficit/Hyperactivity Disorder symptoms in college students. Psychiatr. Q, 89(1), 103-110.

[29] Hannay HJ, Ciaccia PJ, Kerr JW and Barrett D. (1990). Self-report of right-left confusion in college men and women. Percept. Mot. Skills, 70, 451-457.

[30] Malusi BM, Mungai Ch and Odiemo L. (2015). Left-handedness as an overlooked special learning need. Integr. J. $\mathrm{Br}, 2(4)$ 71-89.

\section{How to cite this article}

Makashvili M, Kokrashvili E, Kopadze T, Enukidze G and Abuladze N. (2020). Left-handers, retrained left-handers and right-handers: A comparative study. World Journal of Advanced Research and Reviews, 7(1), 41-47. 